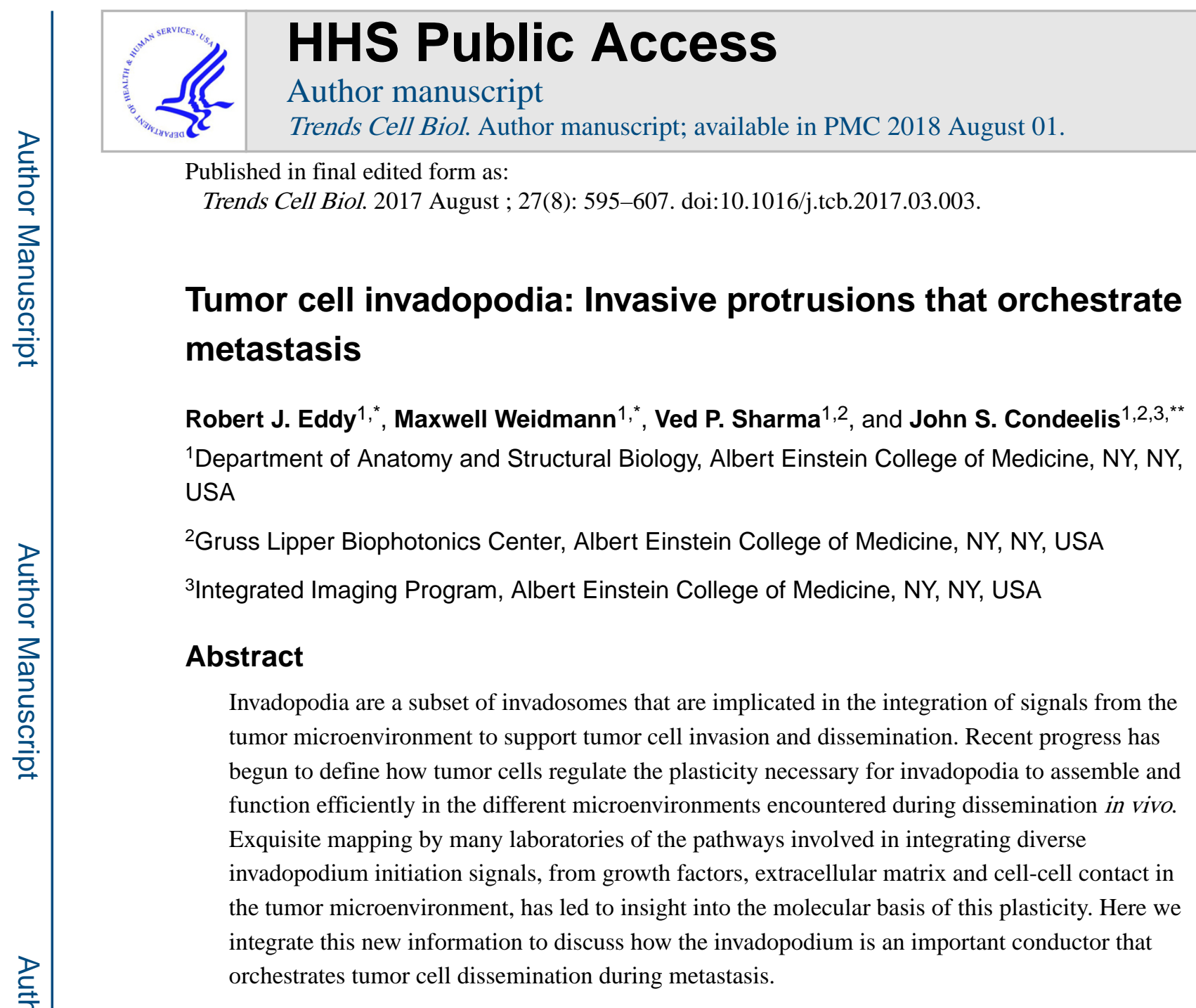

Keywords

Invadopodium-related prognostics; TMEM; Mena; common invadopodium core

\title{
Invadopodia in Cancer
}

The advent of high-resolution intravital imaging of tumors coupled with microarray-based expression profiling of migratory and invasive tumor cells has revealed that oncogenic mutations in tumor cells that drive tumor initiation result in tumor microenvironments that determine tumor cell phenotypes leading to metastasis. The major conductor that orchestrates these tumor cell phenotypes is the invadopodium (see Glossary). In this regard, the invadopodium is a convergence point for the many signals that regulate these tumor cell behaviors. Hence, the invadopodium is a hallmark of tumor cells that undergo systemic dissemination and metastasis.

\footnotetext{
**Corresponding author: John S. Condeelis, John.condeelis@einstein.yu.edu.

*Equal contribution

Publisher's Disclaimer: This is a PDF file of an unedited manuscript that has been accepted for publication. As a service to our customers we are providing this early version of the manuscript. The manuscript will undergo copyediting, typesetting, and review of the resulting proof before it is published in its final citable form. Please note that during the production process errors may be discovered which could affect the content, and all legal disclaimers that apply to the journal pertain.
} 
In this context, it is appropriate to distinguish tumor cell invadopodia from structures broadly defined as invadosomes because tumor cell invadopodia are increasingly implicated in playing highly specific roles in the tumor metastasis which are different from the physiological functions of other invadosomes. Therefore, increasing the resolution of the methods we use to measure, identify and observe invadopodia on tumor cells in vitro and in vivo, and the terminology associated with these efforts, is timely and necessary if we are to design tumor cell specific inhibitors of metastasis (Box 1).

\section{Text box 1}

\section{Invadosomes vs invadopodia}

The term "invadosome" includes a broad category of cell surface structures with proteolytic activity $[4,79]$. Invadosomes have been described in a variety of normal cell types, including osteoclasts [80], macrophages [79], endothelial cells [81] and neurons [82] in normal physiological contexts, and in tumor cells [79]. The term "invadosome" also includes macrophage podosomes and tumor cell invadopodia but these have welldocumented differences in structure, molecular pathways, and functions. Unlike podosomes which are very short lived (minutes) and not protrusive, invadopodia can last for hours as long protrusive structures many microns in length with an F-actin-filled core, the polymerization of which is responsible for invadopodium protrusion. Cartoons summarizing the structural elements of invadopodia are available in Linder et al [79]. Significant differences in molecular properties between podosomes and invadopodia include differences in the expression of key regulatory proteins such as Nck1 and Mena in tumor cell invadopodia but not macrophage podosomes, and WASP and Grb2 in podosomes but not invadopodia [4, 16, 75]. Differences in function have also been identified with invadopodia being uniquely involved in chemotaxis, locomotion and extending long protrusions through tight barriers between cells, small pores in ECM and basement membranes in vitro and in vivo [5, 48]. As our understanding of the role invadopodia play in cancer metastasis increases, it is appropriate to distinguish tumor cell invadopodia from other invadosomes because tumor cell invadopodia are increasingly implicated in playing highly specific roles in integrating signals from the tumor microenvironment. These signals specify tumor cell phenotype in vivo, leading to systemic dissemination and seeding of metastatic tumors in various organ systems [25, 59]. Understanding what is unique about tumor cell invadopodia will allow the development of strategies to selectively inhibit their functions in metastasis without disrupting the normal homeostatic functions of other invadosomes.

Metastasis of breast cancer requires the dissemination of primary tumor cells to distant organs and involves a set of specific cell behaviors in a diverse set of tissue microenvironments. In order to reach distant organs, disseminating tumor cells escape from the primary tumor in several steps. Initially, tumor cells must penetrate the basement membrane of their home epithelium, followed by migration along extracellular matrix (ECM) features of the tumor stroma. Encountering a blood vessel, tumor cells must penetrate the basement membrane of the vascular endothelium and move through the opening between adjacent endothelial cells, a process called transendothelial migration. 
Tumor cells also invade lymphatic vessels which lie outside of the primary breast tumor mass so the involvement of invadopodia in this process is less well understood [1].

Invadopodia have been implicated in the above steps in tumor metastasis [2]. Invadopodia (Box 1) are ideally designed as long protrusions with proteolytic activity to breach the crosslinked networks of ECM that characterize basement membranes of epithelia and endothelia, which restrict tumor cell motility to such a degree that proteolysis is necessary for tumor cell invasion of these structures, and then to cross epithelia and endothelia cell layers [3-5].

\section{Molecular Mechanisms of Invadopodium Initiation and Function}

\section{Stages of invadopodium formation}

Current models derived from studies of adenocarcinoma cells propose that invadopodia form in discrete temporal stages, the definitions of which have evolved as the spatial and temporal resolution of analysis of invadopodium formation has increased (Figure 1). These stages are: invadopodium precursor core initiation, invadopodium precursor stabilization, and invadopodium maturation [6]. Invadopodia initiated by either growth factor or ECM signals can occur in different locations within the same tumor cell [7] or in different tumor cells in different tissue locations [5]. The above stages appear to be the same for these two forms of initiation.

Core initiation involves the recruitment of at least N-WASp, Arp2/3 complex and cofilin around an actin-cortactin complex. This occurs rapidly on a seconds time scale and the resulting precursor core is unstable. Precursor stabilization involves Tks5-mediated anchoring of the precursor core to $\mathrm{PI}(3,4) \mathrm{P} 2$ in the plasma membrane [7-9]. Concerning $\mathrm{PI}(3,4) \mathrm{P} 2$, lamellipodin's $\mathrm{PH}$ domain specifically binds to $\mathrm{PI}(3,4) \mathrm{P} 2$, a lipid that is highly localized to the core compared to other phosphoinositides [7], and recruits Mena to the invadopodium precursor [9]. Lamellipodin also recruits Arg kinase [9]. Since Mena interacts with SHIP2 [8], lamellipodin, therefore, likely recruits a Mena-Arg-SHIP2 complex to the precursor. SHIP2 produces additional PI(3,4)P2 to which Tks5 binds thereby stabilizing the precursor by binding it to the plasma membrane. For the above reason, the co-localization of Tks5 and cortactin uniquely identifies invadopodium precursors. Other combinations of core proteins may not be uniquely associated with the invadopodium core structure during the precursor stage as these proteins are found widely within the actin cytoskeleton of motile cells (Figures 1 and 2).

Stabilization also involves the initiation of new actin polymerization through a synergistic mechanism between cofilin and Arp $2 / 3$ actin polymerization pathways, a step requiring several minutes. In particular, cofilin-mediated actin polymerization generates daughter filaments that activate N-WASp-Arp2/3 complex-mediated nucleation of actin polymerization [10]. Both cofilin and Nck1/N-WASP have been shown to initially activate Arp2/3 in the invadopodium precursor core, suggesting that the role of Cdc42 is to maintain activation of Arp $2 / 3$ in the mature invadopodium [6,11]. Finally, invadopodium maturation follows with core associated actin polymerization to elongate the invadopodium to form a protrusion, MMP recruitment and ECM degradation $[3,6,7]$. 


\section{Anchoring as a key step}

Arguably, a key step in supporting stabilization of the precursor is Tks5-mediated anchoring of the precursor core to the plasma membrane (Figure 2). Tks5, a PI(3,4)P2 and N-WASpbinding protein [3], and SHIP2, a phosphatase that produces PI(3,4)P2 [7], have been implicated in this anchoring step. SHIP2 interacts with a number of molecules including intersectin, Mena and RhoA $[8,12,13]$. SHIP2 sequence contains four canonical N-terminal Mena EVH1-binding motifs consistent with the finding that endogenous SHIP2 binds to Mena $[8,9]$.

\section{RhoGTPases in invadopodium formation and function}

In addition to the central role of CDC42 in actin polymerization above, RhoA and RhoC play essential roles in invadopodium function $[14,15]$. The contact between a macrophage and tumor cell increases RhoA activity in the tumor cell [14] and also causes a dramatic Notch1-dependent increase in Mena ${ }^{\mathrm{INV}}$ expression [16]. The RhoA and Mena changes are noteworthy since Mena is required for RhoA activation in hepatocellular carcinoma cell lines [17]. Furthermore, in glioblastoma [18] Mena binds to Rac1 and inhibits its activity, consistent with other findings that Rac1 inhibition prevents, and Rac1 activation induces, the disassembly of invadopodia [19]. In addition, both RhoA and Cdc42 regulate matrix degradation through a mechanism that involves delivery of MT1-MMP to the invadopodium which activates local matrix degradation [20]. In addition, the spatiotemporal activation dynamics of RhoC have been shown to regulate invadopodium shape and elongation by focusing cofilin's actin polymerization activity into a core-associated, rod-shaped compartment [15].

\section{Mena: a key regulatory protein in invadopodium formation and function}

One of the alternatively spliced isoforms of Mena, Mena ${ }^{\mathrm{INV}}$, is highly upregulated in invasive and disseminating tumor cells [21]. Mena ${ }^{\mathrm{INV}}$ expression potentiates invadopodium activity by sensitizing growth factor receptor and ECM signals by its ability to inhibit PTP1B, a tyrosine phosphatase of c-Met, EGFR and cortactin [8, 22]. Of particular significance are two recent findings; 1) that macrophage contact with tumor cells, as occurs in and around TMEM (Box 2), initiates Mena ${ }^{\mathrm{INV}}$ expression through NOTCH-signaling dependent transcription [16] and 2) that expression of Mena ${ }^{\mathrm{INV}}$ greatly stimulates invadopodium assembly and function in tumor cells by enhancing the phosphorylation of cortactin Y421, a well-known regulatory step in initiating invadopodium formation in invasive tumor cells [22](Figures 1 and 2). Mena ${ }^{\mathrm{INV}}$ promotes phosphorylation of cortactin at tyrosine 421 by displacing PTP1B from the invadopodium core [22]. In preventing the localization of PTP1B to invadopodia, Mena ${ }^{\mathrm{INV}}$ can sensitize cells to a wide range of extracellular stimuli and driver mutations in the tumor cell that promote invadopodium maturation by a common mechanism of cortactin phosphorylation [6, 22](Figures 1 and 2) (Text Box 3). 


\section{Text box 2}

\section{Invadopodium-associated prognostics of breast cancer metastasis}

In breast tumors, tumor cells undergo streaming migration toward blood vessels accompanied by macrophages, and intravasate at microanatomic sites called TMEM

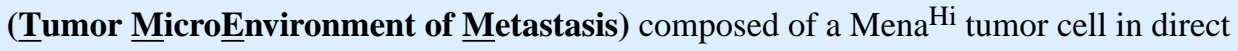
contact with a proangiogenic macrophage and endothelial cell, where tumor cells intravasate $[2,75,83]$. Gene expression profiling of the streaming and intravasating tumor cells identified the Invasion Signature [72, 84] and its associated signaling pathways activated in streaming and intravasating tumor cells [72, 83, 84]. These pathways involve differential expression of Mena isoforms, resulting from alternative splicing, that encode functionally distinct proteins that are expressed in different tumor cells during metastasis $[21,85]$.

The Invasion Signature, and experiments testing its predictions, implicate the Mena expression pattern Mena ${ }^{\text {Classic-Hi }}{ }^{\text {, Mena }}{ }^{\mathrm{INV}-\mathrm{Hi}}$ and Mena11 $\mathrm{a}^{\mathrm{Lo}}$, scored as MenaCalc [86] in regulating TMEM-mediated dissemination of breast tumor cells in vivo [72]. Mena ${ }^{I N V-H i}$ expressing tumor cells have greatly enhanced invadopodium assembly, streaming, TMEM association and intravasation activities, while Mena11a-expressing cells have these phenotypes suppressed [2, 22, 73, 74, 87]. Consistent with these findings in mice, in human breast tumors, TMEM and MenaCalc scores are prognostic for metastasis in breast cancer patients $[70,86]$ and add complimentary information to other currently used prognostics that are based on proliferation alone [72].

TMEM, MenaCalc and Mena ${ }^{\mathrm{INV}}$ are mechanistically linked at the invadopodium. Mena $^{\text {INV }}$ expression increases tumor cell motility, invadopodium assembly and invasion, and potentiates chemotactic responses to EGF and HGF $[8,22,88]$ and haptotactic responses to fibronectin [89]. Mena ${ }^{\mathrm{INV}}$ and its associated invadopodium, are required for TMEM-dependent transendothelial migration of tumor cells from patient biopsies and are necessary for intravasation of all subtypes of breast cancer cells [16, 73].

\section{Text box 3}

\section{The effects of driver mutations are subject to regulatory signals from the tumor microenvironment}

Recent progress has supplied insights into how different signals from the tumor microenvironment initiate the assembly of invadopodium plasticity necessary for invadopodia to assemble and function efficiently in very different microenvironments. The pathways involved in initiation of invadopodia by growth factor receptors, ECM and cell-cell contact have been defined in sufficient detail to allow the creation of models that can be used to guide the investigation of how the tumor microenvironment determines invadopodium phenotype independently of underlying driver mutations. Here, we give one example related to Figures 1 and 2. Driver mutations that lead to constitutive activation of PI3K, Src and Ras, common mutations in many adenocarcinoma, require that homeostatic enzymatic activities in pathways downstream from the driver mutation 
be suppressed in order for the phenotype associated with the driver mutation to emerge. It is known that signals from the tumor microenvironment induce Mena ${ }^{\mathrm{INV}}$ expression in a subpopulation of tumor cells [16]. In preventing the localization of PTP1B to invadopodia, Mena ${ }^{\mathrm{INV}}$ can sensitize this subset of tumor cells to signals from the driver mutations that promote invadopodium maturation by a common mechanism of cortactin phosphorylation (Figure 2). However, in the absence of Mena ${ }^{\mathrm{INV}}$ expression the dephosphorylation of cortactin is fast enough to suppress the driver mutation phenotype which restrains the invasive phenotype to the subset of Mena ${ }^{\mathrm{INV}}$ expressing tumor cells $[6,22]$. In this way the tumor microenvironment determines the invasive behavior of certain tumor cells and, thereby, the malignant phenotype of the tumor [30].

Furthermore, Mena, which can form tetramers potentially of mixed Mena isoform composition, has also been shown to bind to a 5 integrin to regulate interactions with the ECM [23]. Thus, Mena is uniquely positioned to integrate diverse stimuli from the tumor stroma that mediate invasion and dissemination from the primary tumor (Table 1). Since invadopodia are necessary for invasive migration and intravasation by tumor cells [24, 25], the recent results about Mena ${ }^{\mathrm{INV}}$ are consistent with the successful clinical use of the Mena prognostics described in Box 2 and the finding that knockout of the Mena gene inhibits dissemination and metastasis in mouse mammary tumors due to decreased intravasation [26].

\section{Diverse Stimuli Initiate Invadopodium Formation in Diverse Microenvironments}

An important emerging issue in the metastasis field is how do tumor cells regulate the plasticity necessary for invadopodia to assemble and function efficiently in very different microenvironments. The propensity of tumor cells to form invadopodia is driven in part by driver mutations that are responsible for tumor initiation and maintenance. For example, Src and Ras transformation induce invadopodium precursor formation [27, 28]. Activated K-ras can induce pancreatic carcinoma cells to form invadopodia, and cell lines expressing activated K-ras are dependent on expression of this mutant K-ras for invadopodium formation [27]. TGF- $\beta$-induced transformation and induction of EMT by Twist 1 can also stimulate invadopodium formation in epithelial cells [28, 29]. However, there is a growing consensus that these underlying driver mutations are not sufficient to determine tumor phenotype or the behavior of tumor cells in vivo. Rather, the evidence indicates that phenotype is determined by the tumor microenvironment (Text Box 3) [30].

Notwithstanding driver mutations, invadopodia form in response to a wide range of microenvironmental signals, including hypoxia, growth factors, ECM, and cell-cell contact (Table 1). Each of these signals initiates invadopodium assembly in different ways and generates invadopodia with potentially different physiological functions. Different physiological functions would include the function of tumor cell invadopodia in microenvironments relatively devoid of ECM, such as during dissemination from DCIS and extravasation of blood born tumor cells at distant organs, in microenvironments with ECM such as during invasion and migration through dense ECM tissue including basement 
membranes, and in transendothelial migration such as during intravasation of tumor cells in contact with macrophages in primary tumors.

\section{Tumor hypoxia}

The rapid growth of tumors can outpace their available blood supply, creating oxygendeprived (hypoxic) areas within the tumor microenvironment. Tumor hypoxia, regulated by the transcription factor HIF-1a, influences tumor growth and metastasis in a variety of tumor types [31, 32]. In head and neck, lung and pancreas cancer cell lines, HIF-1a is required for hypoxia-induced invadopodium formation through activation of Notch signaling that stimulates ADAM12 activity, a metalloprotease that sheds surface growth factors, leading to increased release of HB-EGF which stimulates invadopodium formation (Figure 2) [33]. Tumors derived from MDA-MB-231 breast carcinoma cells expressing a novel hypoxia reporter reveal an enrichment of hypoxic cells in tumor microenvironments associated with increased chemotaxis toward EGF, enrichment in invadopodia, collagen degradation and intravasation activity [25, 34].

Recent work has confirmed the connection between reactive oxygen species (ROS) production by the NADPH oxidase (Nox) system and invadopodium formation [35], demonstrating how hypoxic conditions may stimulate invadopodium formation by increasing metabolic stress. These results indicate that tumor microenvironments produced by hypoxia trigger invadopodium formation but the relationship of the physiological function of hypoxia-initiated invadopodia to that of invadopodia initiated by the other mechanisms described next remains a topic for future research.

\section{Growth factors}

Stimulation with various growth factors can initiate invadopodium formation. While epidermal growth factor (EGF) is the best characterized growth factor stimulus (Figure 2), transforming growth factor-beta (TGF- $\beta$ ), platelet-derived growth factor (PDGF), hepatocyte growth factor (HGF) and heparin binding EGF (HB-EGF) among others (Table 1) have been shown to induce invadopodium precursor formation in different tumor cell types [28, $29,33,36,37]$. EGF is a necessary component in serum-induced invadopodium assembly in adenocarcinoma cells and is sufficient to stimulate invadopodium formation in the absence of serum (Table 1). In addition, stimulation of a series of mouse mammary tumor cell lines by the soluble growth factor PDGF induces degradation-competent invadopodia and is dependent on the transcription factor Twist1, a key regulator of EMT (Epithelial Mesenchymal Transition) [29]. This ties invadopodium initiation to EMT. Furthermore, tumor cells exposed to an EGF gradient preferentially form invadopodia on the side of cells facing the gradient and these invadopodia are required for chemotactic sensing [2]. This ties invadopodium initiation to chemotaxis and directed cell migration. EMT and directed cell migration are phenotypes associated with disseminating tumor cells [2, 28, 38] suggesting an association between the pathways initiating invadopodium formation with essential steps in the systemic spread of tumors [25, 31-35]. 


\section{Extracelllular matrix}

In the tumor microenvironment, the ECM serves as both a structural substrate and a signaling surface sending information about its composition, density and topology to cells in contact with it. Invadopodium maturation is initiated by extracellular matrix (ECM) through the activity of specific cell-surface adhesion receptors such as integrins and CD44 (Figure 2) (Table 1) [39].

Integrins-Integrin-mediated ECM adhesion is necessary for invadopodium maturation, and the integrins $a 2, a 3, a 5, a 6$ and $\beta 1$, but not $\beta 3$, are localized to invadopodia $[6,40,41]$. Out of the 23 distinct integrin heterodimers that have been observed, the localization of four specific integrin receptors $(\alpha 2 \beta 1, \alpha 3 \beta 1$, a $5 \beta 1$ and $\alpha 6 \beta 1)$ at invadopodia suggests that invadopodia respond selectively to specific components of the ECM [6, 41-43]. The ligand specificity of integrin receptors is determined mainly by the a-subunit, and ECM ligands known to activate these integrin receptors present at invadopodia include fibronectin, type I collagen, and laminin [3, 6, 40, 43]. Fibronectin and a 5 $\beta 1$, in particular, have been associated with increased tumor invasion and poor patient prognosis [6, 40, 44, 45]. Additionally, laminin secretion has been found to suppress invadopodium formation, and inhibition of the laminin receptor, a $3 \beta 1$ integrin, promoted invadopodium formation [3].

In tumor cell invadopodia, $\beta 1$-integrin has been shown to play a particularly important role in promoting invadopodium maturation through its effect on Arg kinase, which is responsible for cortactin phosphorylation at specific tyrosines (Y421 and Y466)(Figure 2). These tyrosines are necessary for invadopodium maturation and the steps of maturation, have been defined as discussed above $[10,36,40,46]$. $\beta 1$-integrin is able to directly interact with Arg via its cytoplasmic tail, and this interaction promotes Arg kinase activity toward cortactin Y421 and Y466 (Figures 2) [36, 47].

$\beta 1$-integrin also participates in the recruitment of talin- and moesin to the invadopodium core leading to the attachment of the sodium-hydrogen antiporter NHE1 to invadopodia [44] (Figure 2). The activity of NHE1 is associated with acidification of the extracellular space and increased $\mathrm{pH}$ in the intracellular space in the invadopodium core leading to activation of cofilin-dependent actin polymerization at invadopodia [44, 48]. In addition, decreased extracellular $\mathrm{pH}$ promotes the delivery of soluble (MMP2 and MMP9) and insoluble (MT1MMP) proteases to invadopodia [49]. These changes in $\mathrm{pH}$ in tumors are associated with tumor progression [50].

Focal adhesion kinase (FAK) regulates invadopodium formation by sequestration of active Src kinase at focal adhesions, and normal FAK activation has been shown to depend on Mena binding to a $5 \beta 1$ integrin [23]. The direct and indirect effects of adhesion proteins on invadopodium assembly are highlighted by observations that invadopodia tend to preferentially initiate at sites of ECM adhesion [7]. Mena, which is present in both focal adhesions and invadopodia, may regulate the relationship between these two domains through its interaction with a $5 \beta 1$ integrin present at both locations [22, 23].

CD44-CD44 is a commonly used cancer stem cell marker which is associated with the subpopulation of invadopodium containing migrating and disseminating tumor cells in 
breast tumors [5, 51, 52]. CD44 enables invasive tumor cells to produce functional invadopodia in response to metastasis-promoting microenvironments [52, 53]. Originally characterized as a hyaluronan receptor, CD44 is involved in the adhesion of tumor cells to collagen type-I and -IV, laminin and fibronectin [54]. CD44 is associated with histopathological markers of tumor progression and disease recurrence in multiple types of cancer [53]. Recent work has shown that CD44 promotes a $5 \beta 1$ activation in response to fibronectin [54], which has been shown to promote invadopodium maturation [40]. CD44 has been shown to be in complex with MMP9 at invadopodia in breast cancer cells, and is involved with MMP9 recruitment to the ventral cell surface of lymphoma cells [55].

Furthermore, a specific splice-isoform of CD44 associated with metastasis and necessary for EMT also enhances invadopodium maturation, increasing cortactin phosphorylation at Y421 and MT1-MMP recruitment to invadopodia [52] (Figure 2).

ECM Modification-After the disassembly of the invadopodium core proteins from the site of a mature invadopodium, MT1-MMP has been observed to remain where ECM degradation occurred [56]. This raises the possibility that tumor cells may use invadopodia to deposit membrane-bound MMPs, which have adhered to the degraded ECM substrate, and thereby mark sites of future invadopodium formation and ECM degradation as occurs in podosomes [57].

Another factor in self-reinforced invadopodium activation is the breakdown of the tumor ECM by invadopodium-associated MMP activity which releases numerous bioactive protein fragments such as the laminin-111-derived peptides AG73 and C16 that are capable of increasing invadopodium formation in a $\beta 1$ integrin-dependent manner in tumor cells [58]. Such bioactive molecules released by MMP activity may serve to identify sites throughout the tumor that have been visited by other tumor cells, identifying these sites as places that offer less resistance to migration, and places that initiate invadopodia independently of other signals.

Matrix rigidity can also stimulate invadopodium maturation [59,60]. This enhancement of invadopodium maturation is dependent on the acto-myosin contractile apparatus, suggesting that invading tumor cells respond to more than just chemical cues encountered in the ECM, and that the actin cytoskeleton dynamically adapts to changes in matrix rigidity that require proteolysis for tumor cell migration [59, 61]. The effect of ECM rigidity on invadopodium maturation is mediated by the different effects of Rho-associated kinase ROCK1/2 [62]. ROCK1/2 accomplishes this by regulating both the acto-myosin contractile apparatus via non-muscle myosin II activation, and direct regulation of LIM kinase's phosphorylation of cofilin to spatially couple Rho-G protein activity to the activation of cofilin-induced actin polymerization in the invadopodium core $[10,15,63]$, leading to invadopodium maturation and modulation of invadopodium shape [62-64] and turnover [19, 48].

The above considerations indicate mechanisms that provide plasticity to invadopodia to sculpt their morphology to suit the particular matrix they encounter independently of driver mutations [39] (Box 3). These specially positioned and shaped invadopodia coordinate invadopodium function including the cylindrical shaping of invadopodia by the spatial regulation of RhoC activation [15], and the response of tumor cells to fibrillar type-I 
collagen which induces the formation of linear invadopodia [65, 66]. These linear invadopodia require the activity of $\mathrm{Cdc} 42$ and Tuba, the latter of which is able to detect deformations in the matrix with its F-BAR domain $[65,66]$. In addition, high-density fibrillar collagen matrix substrate (HDFC) can induce invadopodia in breast tumor cells by engagement of a specific a $2 \beta 1$ integrin signaling pathway that does not require growth factor stimulation or alterations in gene and protein expression [41].

\section{Exosomes}

The secretion of exosomes promotes invadopodium formation and maturation, as well as in vitro invasion of head and neck squamous cell carcinoma, by facilitating the exocytosis of MT1-MMP matrix-degrading proteinase at sites of actively degrading mature invadopodia [67]. The addition of purified exosomes to SCC61 cells increases the number of invadopodia and extends invadopodium lifetimes in the absence of growth factors. Interestingly, while EGF induction of invadopodia is rapid, induction by exogenous exosomes is far slower, taking up to an hour suggesting the use of different or additional stages in invadopodium maturation between these two different initiation signals. These data suggest a unique role for exosomes and their cargoes in promoting invasive activity through invadopodium formation independent of growth factor stimulation [67].

\section{Stromal cell-tumor cell contact}

Tumor cells make prolonged direct contact with some stromal cells during dissemination from the primary tumor. The best characterized of these direct contacts is that with immune cells, macrophages and neutrophils, in particular. Of relevance here, these contacts have a role in inducing invadopodium formation and invadopodium-dependent intravasation during tumor cell dissemination $[14,16,68,69]$. One of the most carefully studied and clinically relevant events involving the direct contact between a tumor cell and immune cell during metastasis is the direct contact of a tumor cell with a proangiogenic macrophage where both cells are also in contact with an endothelial cell on the surface of a blood vessel [70-72]. This three-cell complex is called the Tumor Microenvironment of Metastasis (TMEM) and uses macrophage-induced tumor cell invadopodia to support vascular permeability and transendothelial migration by disrupting endothelial cell-cell adhesion resulting in tumor cell intravasation (Box 2) [14, 73-75]. The discovery of TMEM is clinically significant since the number of TMEM in histological samples from breast cancer patients is an independent prognostic indicator of metastatic recurrence $[70,73,74]$ and has been validated for use with ER+ breast cancer patients [70,72]. High-resolution in vitro and in vivo imaging of TMEM function reveals that macrophage-tumor cell contact induces Notch1 signaling resulting in RhoA-and Mena-dependent invadopodium formation in the TMEM tumor cell (Figure 2). Furthermore, macrophage-driven increases in tumor cell intravasation were dependent on this contact-induced invadopodium $[14,16]$. These results are consistent with the requirement for invadopodium function for intravasation and dissemination of tumor cells in mammary tumors $[24,25]$ and may underlie the prognostic significance of TMEM in breast cancer patients (Box 2).

Since invadopodia have been found to enhance exosome secretion [67], and exosome secretion into the bloodstream contributes to formation of the pre-metastatic niche [76, 77], 
induction of invadopodium formation at TMEM sites suggests a mechanism by which Mena ${ }^{\mathrm{INV}}$-containing invadopodia contribute to metastasis by enabling tumor cells to supply exosomes to the vasculature that promote formation of the pre-metastatic niche at distant sites [67, 76-78].

\section{Concluding Remarks}

Recent work has resolved invadopodium assembly and maturation into distinct stages.

However, the signaling inputs and microenvironments that promote invadopodium assembly and maturation have not yet been studied sufficiently to define the specific stages they each regulate (see Outstanding Questions). By employing markers that identify invadopodia at distinct stages of assembly and maturation, and techniques with high temporal and spatial resolution, including high-resolution intravital microscopy, biosensors of pathway activity at subcellular resolution in vivo, and the detection of gene expression patterns in single cells in situ by high-resolution FISH, future work will be able to address the specific contributions to invadopodium assembly of each of the initiating signals in the tumor microenvironment.

\section{Outstanding Questions Box}

How can tools with high temporal and spatial resolution be used to fully understand the similarities and differences in the assembly and function of invadopodia initiated on tumor cells by different tumor microenvironments?

Which of the stages of invadopodium assembly and maturation are regulated by each of the initiation signals in the tumor microenvironment?

Do different initiation signals lead to invadopodia with different lifetimes and ECM degradation activities?

What are the markers of invadopodia, specifically involved in tumor metastasis, which can be used as targets for prognosis of distant metastasis and therapeutic inhibition of tumor cell dissemination?

How are the molecular pathways, associated with crosstalk between invadopodia and chemo- and haptotaxis, coordinated during tumor cell dissemination in vivo?

What are the mechanisms underlying exosome-mediated invadopodium initiation?

How does exosome-mediated invadopodium initiation contribute to metastatic seeding at distant sites?

Are invadopodia involved in the same steps of transendothelial migration during intravasation and extravasation?

What are the molecular relationships common among hypoxia, cancer stem cells and invadopodium function?

It is clear from the recent work discussed above that invadopodia play an essential role in transendothelial migration during intravasation and systemic dissemination of tumor cells. However, future work will be required to determine, at subcellular resolution, the specific 
functions of invadopodia during transendothelial migration. For example, are invadopodia involved in the same functions in both the intravasation and extravasation directions of transendothelial migration, and which molecular steps in the stages of invadopodium assembly and maturation are best targets for therapeutic intervention to prevent transendothelial migration-dependent dissemination of tumor cells?

Another general theme to emerge from the recent studies discussed above is the relationships of invadopodium initiation to EMT, and to directed migration of tumor cells in vivo, involving chemotaxis to growth factors and haptotaxis on ECM structures. All of these events contribute to systemic tumor cell dissemination so understanding their molecular crosstalk is essential. Future work at the molecular level will be needed to define, with precision, the mechanisms and molecular pathways involved in this crosstalk.

Increasingly, hypoxia is associated with invadopodium-containing tumor cells. In addition, some cancer stem cell markers have been implicated in invadopodium assembly and function. It is important to remember that the dissemination of cancer stem cells is an essential step in metastasis and tumor cells require invadopodia for dissemination. Future work will be needed to define the relationships between hypoxia, stemness and invadopodium function, including exosome secretion, at the molecular level before we can hope to understand metastasis at the level required to prevent it.

\section{Glossary}

\section{Alternative Splicing}

Post-transcriptional modification of mRNA by splicing factors to change the exon or intron composition of the mRNA transcript, thereby enabling a single gene to produce different protein products (isoforms).

\section{Basement membrane}

Specific type of extracellular matrix produced by an epithelium at its basal surface, and typically rich in dense and highly cross-linked type IV collagen and laminin.

\section{Cytoskeleton}

An intracellular protein scaffold composed of several types of filament polymers, including actin microfilaments, intermediate filaments (e.g. keratin), and microtubules (tubulin). Maintains cell structure, mediates protein trafficking, and generates the forces for cell motility and cell division.

\section{Dissemination}

Spread of tumor cells to adjacent tissues, organs or nonadjacent organs, either by local migration or the circulation, prior to colonization.

\section{Epithelium}

Single (simple) or multiple (stratified) layer(s) of cells that form a boundary between two tissue compartments.

\section{Endothelium}


Type of epithelial monolayer of flat (squamous) cells that line blood and lymphatic vessels.

\section{Extracellular matrix (ECM)}

Network of insoluble molecules secreted by cells that create structural support for tissues and provide a scaffold for cell migration and signaling.

\section{Epithelial to Mesenchymal Transition (EMT)}

A process occurring during embryogenesis and tumor progression involving broad changes in gene expression whereby epithelial cells lose their typical polarity, cell-cell junctions and morphology and adopt migratory phenotypes similar to mesenchymal cells.

\section{Exosomes}

Small vesicles (30-100nm) produced by the budding of multi-vesicular endosomes (MVEs) and released from the cell when MVEs fuse with the plasma membrane. Exosomes carry a wide variety of cell-specific cargo and are principally associated with cell-cell communication.

\section{Growth Factor}

A signaling molecule capable of inducing cell proliferation, typically a protein or steroid hormone.

\section{Invadopodium}

An actin-rich membrane protrusion produced by carcinoma cells that is capable of adhesion to and degradation of the extracellular matrix.

\section{Mena}

Mena [also referred to as ENAH (Enabled homolog) is the mammalian ortholog of Drosphila Enabled (Ena). Alternative splicing produces distinct Mena protein isoforms including Mena ${ }^{\mathrm{INV}}$ which is found exclusively in invasive tumor cells. Mena forms tetramers potentially consisting of mixed isoforms.

\section{Metastasis}

The colonization of non-adjacent organs by tumor cells spread through the blood or lymphatic circulation. Initial colonization is referred to as micrometastasis, whereas proliferation to form a clinically detectible tumor mass is a macrometastasis.

\section{Microenvironment}

The set of factors in the immediate vicinity of tumor cells that influence their phenotype, including chemical signals (e.g. growth factors), mechanical forces from ECM (e.g. rigidity), and cell-cell contact.

\section{Proteolysis}

Cleavage of proteins by enzymatic catalysis of peptide bonds.

\section{References}

1. Podgrabinska S, Skobe M. Role of lymphatic vasculature in regional and distant metastases. Microvasc Res. 2014; 95:46-52. [PubMed: 25026412] 
2. Roussos ET, Condeelis JS, Patsialou A. Chemotaxis in cancer. Nat Rev Cancer. 2011; 11(8):573-87. [PubMed: 21779009]

3. Murphy DA, Courtneidge SA. The 'ins' and 'outs' of podosomes and invadopodia: characteristics, formation and function. Nat Rev Mol Cell Biol. 2011; 12(7):413-26. [PubMed: 21697900]

4. Sibony-Benyamini H, Gil-Henn H. Invadopodia: the leading force. Eur J Cell Biol. 2012; 91(1112):896-901. [PubMed: 22633185]

5. Genot E, Gligorijevic B. Invadosomes in their natural habitat. Eur J Cell Biol. 2014; 93(10-12):36779. [PubMed: 25457677]

6. Beaty BT, Condeelis J. Digging a little deeper: the stages of invadopodium formation and maturation. Eur J Cell Biol. 2014; 93(10-12):438-44. [PubMed: 25113547]

7. Sharma VP, et al. Tks5 and SHIP2 regulate invadopodium maturation, but not initiation, in breast carcinoma cells. Curr Biol. 2013; 23(21):2079-89. [PubMed: 24206842]

8. Hughes SK, et al. PTP1B-dependent regulation of receptor tyrosine kinase signaling by the actinbinding protein Mena. Mol Biol Cell. 2015; 26(21):3867-78. [PubMed: 26337385]

9. Carmona $\mathrm{G}$, et al. Lamellipodin promotes invasive 3D cancer cell migration via regulated interactions with Ena/VASP and SCAR/WAVE. Oncogene. 2016; 35(39):5155-69. [PubMed: 26996666]

10. Bravo-Cordero JJ, et al. Functions of cofilin in cell locomotion and invasion. Nat Rev Mol Cell Biol. 2013; 14(7):405-15. [PubMed: 23778968]

11. Helgeson LA, Nolen BJ. Mechanism of synergistic activation of Arp2/3 complex by cortactin and N-WASP. Elife. 2013; 2:e00884. [PubMed: 24015358]

12. Nakatsu F, et al. The inositol 5-phosphatase SHIP2 regulates endocytic clathrin-coated pit dynamics. J Cell Biol. 2010; 190(3):307-15. [PubMed: 20679431]

13. Kato K, et al. The inositol 5-phosphatase SHIP2 is an effector of RhoA and is involved in cell polarity and migration. Mol Biol Cell. 2012; 23(13):2593-604. [PubMed: 22593208]

14. Roh-Johnson M, et al. Macrophage contact induces RhoA GTPase signaling to trigger tumor cell intravasation. Oncogene. 2014; 33(33):4203-12. [PubMed: 24056963]

15. Bravo-Cordero JJ, Hodgson L, Condeelis J. Directed cell invasion and migration during metastasis. Curr Opin Cell Biol. 2012; 24(2):277-83. [PubMed: 22209238]

16. Pignatelli J, et al. Macrophage-dependent tumor cell transendothelial migration is mediated by Notch1/MenaINV-initiated invadopodium formation. Sci Rep. 2016; 6:37874. [PubMed: 27901093]

17. Lin L, et al. Microfilament regulatory protein MENA increases activity of RhoA and promotes metastasis of hepatocellular carcinoma. Exp Cell Res. 2014; 327(1):113-22. [PubMed: 24859350]

18. Higashi M, et al. Human Mena associates with Rac1 small GTPase in glioblastoma cell lines. PLoS One. 2009; 4(3):e4765. [PubMed: 19277120]

19. Moshfegh Y, et al. A Trio-Rac1-Pak1 signalling axis drives invadopodia disassembly. Nat Cell Biol. 2014; 16(6):574-86. [PubMed: 24859002]

20. Jacob A, Prekeris R. The regulation of MMP targeting to invadopodia during cancer metastasis. Front Cell Dev Biol. 2015; 3:4. [PubMed: 25699257]

21. Gertler F, Condeelis J. Metastasis: tumor cells becoming MENAcing. Trends Cell Biol. 2011; 21(2):81-90. [PubMed: 21071226]

22. Weidmann MD, et al. MenaINV dysregulates cortactin phosphorylation to promote invadopodium maturation. Sci Rep. 2016; 6:36142. [PubMed: 27824079]

23. Gupton SL, et al. Mena binds alpha5 integrin directly and modulates alpha5beta1 function. J Cell Biol. 2012; 198(4):657-76. [PubMed: 22908313]

24. Gligorijevic B, et al. N-WASP-mediated invadopodium formation is involved in intravasation and lung metastasis of mammary tumors. J Cell Sci. 2012; 125(Pt 3):724-34. [PubMed: 22389406]

25. Gligorijevic B, Bergman A, Condeelis J. Multiparametric classification links tumor microenvironments with tumor cell phenotype. PLoS Biol. 2014; 12(11):e1001995. [PubMed: 25386698] 
26. Roussos ET, et al. Mena deficiency delays tumor progression and decreases metastasis in polyoma middle-T transgenic mouse mammary tumors. Breast Cancer Res. 2010; 12(6):R101. [PubMed: 21108830]

27. Neel NF, et al. The RalB small GTPase mediates formation of invadopodia through a GTPaseactivating protein-independent function of the RalBP1/RLIP76 effector. Mol Cell Biol. 2012; 32(8):1374-86. [PubMed: 22331470]

28. Pignatelli J, et al. Hic-5 promotes invadopodia formation and invasion during TGF-beta-induced epithelial-mesenchymal transition. J Cell Biol. 2012; 197(3):421-37. [PubMed: 22529104]

29. Eckert MA, et al. Twist1-induced invadopodia formation promotes tumor metastasis. Cancer Cell. 2011; 19(3):372-86. [PubMed: 21397860]

30. DeClerck YA, et al. The Tumor Microenvironment at a Turning Point Knowledge Gained Over the Last Decade, and Challenges and Opportunities Ahead: A White Paper from the NCI TME Network. Cancer Res. 2017

31. Zhang H, et al. HIF-1-dependent expression of angiopoietin-like 4 and L1CAM mediates vascular metastasis of hypoxic breast cancer cells to the lungs. Oncogene. 2012; 31(14):1757-70. [PubMed: 21860410]

32. Lucien F, et al. Hypoxia-induced invadopodia formation involves activation of NHE-1 by the p90 ribosomal S6 kinase (p90RSK). PLoS One. 2011; 6(12):e28851. [PubMed: 22216126]

33. Diaz B, et al. Notch increases the shedding of HB-EGF by ADAM12 to potentiate invadopodia formation in hypoxia. J Cell Biol. 2013; 201(2):279-92. [PubMed: 23589494]

34. Wang Y, et al. Direct visualization of the phenotype of hypoxic tumor cells at single cell resolution in vivo using a new hypoxia probe. Intravital. 2016; 5(2):e1187803. [PubMed: 27790387]

35. Choi JA I, Lim K. TIS21/BTG2 inhibits invadopodia formation by downregulating reactive oxygen species level in MDA-MB-231 cells. J Cancer Res Clin Oncol. 2013; 139(10):1657-65. [PubMed: 23907596]

36. Mader CC, et al. An EGFR-Src-Arg-cortactin pathway mediates functional maturation of invadopodia and breast cancer cell invasion. Cancer Res. 2011; 71(5):1730-41. [PubMed: 21257711]

37. Rajadurai CV, et al. Met receptor tyrosine kinase signals through a cortactin-Gab1 scaffold complex, to mediate invadopodia. J Cell Sci. 2012; 125(Pt 12):2940-53. [PubMed: 22366451]

38. Pang MF, et al. TGF-beta1-induced EMT promotes targeted migration of breast cancer cells through the lymphatic system by the activation of CCR7/CCL21-mediated chemotaxis. Oncogene. 2016; 35(6):748-60. [PubMed: 25961925]

39. Di Martino J, et al. The microenvironment controls invadosome plasticity. J Cell Sci. 2016; 129(9): 1759-68. [PubMed: 27029343]

40. Beaty BT, et al. beta1 integrin regulates Arg to promote invadopodial maturation and matrix degradation. Mol Biol Cell. 2013; 24(11):1661-75. S1-11. [PubMed: 23552693]

41. Artym VV, et al. Dense fibrillar collagen is a potent inducer of invadopodia via a specific signaling network. J Cell Biol. 2015; 208(3):331-50. [PubMed: 25646088]

42. Branch KM, Hoshino D, Weaver AM. Adhesion rings surround invadopodia and promote maturation. Biol Open. 2012; 1(8):711-22. [PubMed: 23213464]

43. Pan L, et al. Research advances on structure and biological functions of integrins. Springerplus. 2016; 5(1):1094. [PubMed: 27468395]

44. Beaty BT, et al. Talin regulates moesin-NHE-1 recruitment to invadopodia and promotes mammary tumor metastasis. J Cell Biol. 2014; 205(5):737-51. [PubMed: 24891603]

45. Bae YK, et al. Fibronectin expression in carcinoma cells correlates with tumor aggressiveness and poor clinical outcome in patients with invasive breast cancer. Hum Pathol. 2013; 44(10):2028-37. [PubMed: 23684510]

46. Oser M, et al. Specific tyrosine phosphorylation sites on cortactin regulate Nck1-dependent actin polymerization in invadopodia. J Cell Sci. 2010; 123(Pt 21):3662-73. [PubMed: 20971703]

47. Simpson MA, et al. Direct interactions with the integrin beta1 cytoplasmic tail activate the Abl2/Arg kinase. J Biol Chem. 2015; 290(13):8360-72. [PubMed: 25694433] 
48. Magalhaes MA, et al. Cortactin phosphorylation regulates cell invasion through a $\mathrm{pH}$-dependent pathway. J Cell Biol. 2011; 195(5):903-20. [PubMed: 22105349]

49. Brisson L, et al. $\mathrm{pH}$ regulators in invadosomal functioning: proton delivery for matrix tasting. Eur J Cell Biol. 2012; 91(11-12):847-60. [PubMed: 22673002]

50. Webb BA, et al. Dysregulated pH: a perfect storm for cancer progression. Nat Rev Cancer. 2011; 11(9):671-7. [PubMed: 21833026]

51. Pattabiraman DR, Weinberg RA. Tackling the cancer stem cells - what challenges do they pose? Nat Rev Drug Discov. 2014; 13(7):497-512. [PubMed: 24981363]

52. Zhao P, et al. The CD44s splice isoform is a central mediator for invadopodia activity. J Cell Sci. 2016; 129(7):1355-65. [PubMed: 26869223]

53. $\mathrm{Xu} \mathrm{H}$, et al. The role of CD44 in epithelial-mesenchymal transition and cancer development. Onco Targets Ther. 2015; 8:3783-92. [PubMed: 26719706]

54. McFarlane S, et al. CD44-mediated activation of alpha5beta1-integrin, cortactin and paxillin signaling underpins adhesion of basal-like breast cancer cells to endothelium and fibronectinenriched matrices. Oncotarget. 2015; 6(34):36762-73. [PubMed: 26447611]

55. Lagarrigue F, et al. Matrix metalloproteinase- 9 is upregulated in nucleophosmin-anaplastic lymphoma kinase-positive anaplastic lymphomas and activated at the cell surface by the chaperone heat shock protein 90 to promote cell invasion. Cancer Res. 2010; 70(17):6978-87. [PubMed: 20699364]

56. Hoshino D, Branch KM, Weaver AM. Signaling inputs to invadopodia and podosomes. J Cell Sci. 2013; 126(Pt 14):2979-89. [PubMed: 23843616]

57. El Azzouzi K, Wiesner C, Linder S. Metalloproteinase MT1-MMP islets act as memory devices for podosome reemergence. J Cell Biol. 2016; 213(1):109-25. [PubMed: 27069022]

58. Siqueira AS, et al. Laminin-111 peptide C16 regulates invadopodia activity of malignant cells through beta1 integrin, Src and ERK 1/2. Oncotarget. 2016; 7(30):47904-47917. [PubMed: 27323814]

59. Parekh A, Weaver AM. Regulation of invadopodia by mechanical signaling. Exp Cell Res. 2016; 343(1):89-95. [PubMed: 26546985]

60. Parekh A, et al. Sensing and modulation of invadopodia across a wide range of rigidities. Biophys J. 2011; 100(3):573-82. [PubMed: 21281571]

61. Jerrell RJ, Parekh A. Cellular traction stresses mediate extracellular matrix degradation by invadopodia. Acta Biomater. 2014; 10(5):1886-96. [PubMed: 24412623]

62. Jerrell RJ, Parekh A. Matrix rigidity differentially regulates invadopodia activity through ROCK1 and ROCK2. Biomaterials. 2016; 84:119-29. [PubMed: 26826790]

63. Bravo-Cordero JJ, et al. A novel spatiotemporal RhoC activation pathway locally regulates cofilin activity at invadopodia. Curr Biol. 2011; 21(8):635-44. [PubMed: 21474314]

64. Scott RW, et al. LIM kinases are required for invasive path generation by tumor and tumorassociated stromal cells. J Cell Biol. 2010; 191(1):169-85. [PubMed: 20876278]

65. Di Martino J, Moreau V, Saltel F. Type I collagen fibrils: an inducer of invadosomes. Oncotarget. 2015; 6(30):28519-20. [PubMed: 26418722]

66. Juin A, et al. Discoidin domain receptor 1 controls linear invadosome formation via a Cdc42-Tuba pathway. J Cell Biol. 2014; 207(4):517-33. [PubMed: 25422375]

67. Hoshino D, et al. Exosome secretion is enhanced by invadopodia and drives invasive behavior. Cell Rep. 2013; 5(5):1159-68. [PubMed: 24290760]

68. Komohara Y, et al. Tumor-associated macrophages: Potential therapeutic targets for anti-cancer therapy. Adv Drug Deliv Rev. 2016; 99(Pt B):180-5. [PubMed: 26621196]

69. Glogauer JE, et al. Neutrophils Increase Oral Squamous Cell Carcinoma Invasion through an Invadopodia-Dependent Pathway. Cancer Immunol Res. 2015; 3(11):1218-26. [PubMed: 26112922]

70. Rohan TE, et al. Tumor microenvironment of metastasis and risk of distant metastasis of breast cancer. J Natl Cancer Inst. 2014; 106(8)

71. Oktay MH, Jones JG. TMEM: a novel breast cancer dissemination marker for the assessment of metastatic risk. Biomark Med. 2015; 9(2):81-4. [PubMed: 25689896] 
72. Karagiannis GS, et al. Signatures of breast cancer metastasis at a glance. J Cell Sci. 2016; 129(9): 1751-8. [PubMed: 27084578]

73. Pignatelli $\mathrm{J}$, et al. Invasive breast carcinoma cells from patients exhibit MenaINV- and macrophage-dependent transendothelial migration. Sci Signal. 2014; 7(353):ra112. [PubMed: 25429076]

74. Roussos ET, et al. Mena invasive (Mena(INV)) and Mena11a isoforms play distinct roles in breast cancer cell cohesion and association with TMEM. Clin Exp Metastasis. 2011; 28(6):515-27. [PubMed: 21484349]

75. Harney AS, et al. Real-Time Imaging Reveals Local, Transient Vascular Permeability, and Tumor Cell Intravasation Stimulated by TIE2hi Macrophage-Derived VEGFA. Cancer Discov. 2015; 5(9): 932-43. [PubMed: 26269515]

76. Costa-Silva B, et al. Pancreatic cancer exosomes initiate pre-metastatic niche formation in the liver. Nat Cell Biol. 2015; 17(6):816-26. [PubMed: 25985394]

77. Hoshino A, et al. Tumour exosome integrins determine organotropic metastasis. Nature. 2015; 527(7578):329-35. [PubMed: 26524530]

78. Kenific CM, Nogues L, Lyden D. Pre-Metastatic Niche Formation Has Taken Its TOLL. Cancer Cell. 2016; 30(2):189-91. [PubMed: 27505667]

79. Linder S, Wiesner C, Himmel M. Degrading devices: invadosomes in proteolytic cell invasion. Annu Rev Cell Dev Biol. 2011; 27(1):185-211. [PubMed: 21801014]

80. Song RL, et al. New roles of filopodia and podosomes in the differentiation and fusion process of osteoclasts. Genet Mol Res. 2014; 13(3):4776-87. [PubMed: 25062413]

81. Seano G, et al. Podosomes as novel players in endothelial biology. Eur J Cell Biol. 2014; 93(1012):405-12. [PubMed: 25199436]

82. Santiago-Medina M, et al. Regulation of ECM degradation and axon guidance by growth cone invadosomes. Development. 2015; 142(3):486-96. [PubMed: 25564649]

83. Patsialou A, et al. Intravital multiphoton imaging reveals multicellular streaming as a crucial component of in vivo cell migration in human breast tumors. Intravital. 2013; 2(2):e25294. [PubMed: 25013744]

84. Patsialou A, et al. Selective gene-expression profiling of migratory tumor cells in vivo predicts clinical outcome in breast cancer patients. Breast Cancer Res. 2012; 14(5):R139. [PubMed: 23113900]

85. Shapiro IM, et al. An EMT-driven alternative splicing program occurs in human breast cancer and modulates cellular phenotype. PLoS Genet. 2011; 7(8):e1002218. [PubMed: 21876675]

86. Forse CL, et al. Menacalc, a quantitative method of metastasis assessment, as a prognostic marker for axillary node-negative breast cancer. BMC Cancer. 2015; 15:483. [PubMed: 26112005]

87. Roussos ET, et al. Mena invasive (MenaINV) promotes multicellular streaming motility and transendothelial migration in a mouse model of breast cancer. J Cell Sci. 2011; 124(Pt 13):2120_ 31. [PubMed: 21670198]

88. Leung E, et al. Blood vessel endothelium-directed tumor cell streaming in breast tumors requires the HGF/C-Met signaling pathway. Oncogene. 2016:1-13.

89. Oudin MJ, et al. Tumor Cell-Driven Extracellular Matrix Remodeling Drives Haptotaxis during Metastatic Progression. Cancer Discov. 2016; 6(5):516-31. [PubMed: 26811325] 


\section{Trends Box}

Invadopodia form with the same precursor core structure in response to a range of signals from the tumor microenvironment, including soluble growth factors, ECM, cell-contact mediated signaling, and hypoxia. These signals determine tumor phenotype notwithstanding driver mutations initiating the tumor.

Different signals lead to invadopodia with different functions in vivo. In addition to their proteolytic function, invadopodia play key roles in sensing the physical properties of the tumor microenvironment, and in transendothelial migration of tumor cells during dissemination leading to metastasis.

In producing localized MMP activity, decreasing stromal $\mathrm{pH}$, and secreting exosomes, invadopodia regulate tumor cell communication over both short and long distances through these modifications of the stromal microenvironment and exosome-mediated establishment of the pre-metastatic niche. 


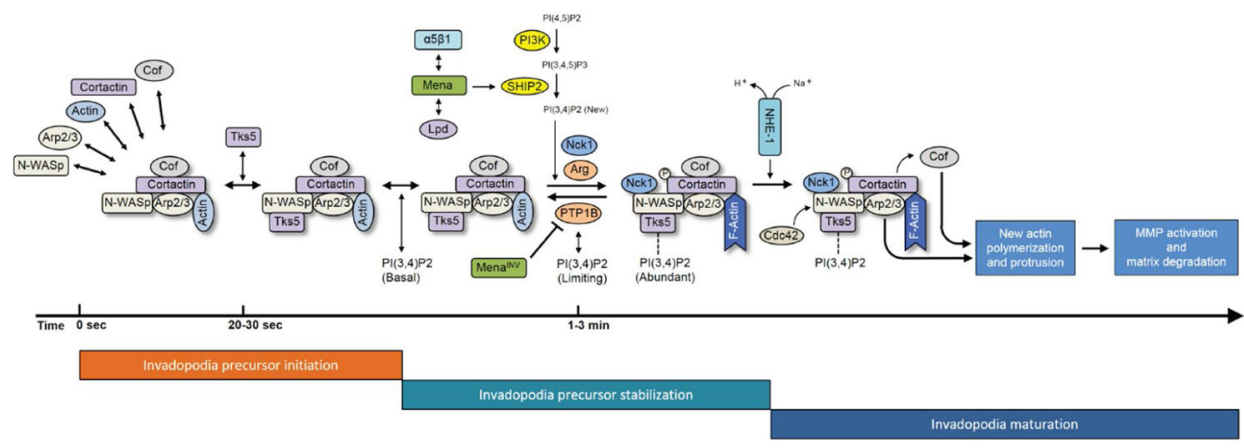

Figure 1. Temporal model of precursor core assembly and maturation common to invadopodium initiation signals

Invadopodium precursors initiate with the arrival of cofilin, Arp2/3, and N-WASp to an actin-cortactin complex. The proportion of G- and F-actin in the precursor at these early stages is unknown. Tks5 arrives $\sim 20$ s later, and this stabilizes the precursor complex by linking it to basal levels of $\mathrm{PI}(3,4) \mathrm{P} 2$ at the plasma membrane. Arg phosphorylation of cortactin at Y421 activates the Nck1-N-WASp complex to stimulate the initial Arp2/3dependent nucleation of actin polymerization which is limited. This leads to recruitment of the moesin-NHE1 complex resulting in the local increase in $\mathrm{pH}$ which activates cofilin. The following 2-3 minutes show both a rapid increase in cofilin-dependent actin polymerization which synergizes with Arp2/3 activity to cause a large increase in new actin polymerization and invadopodium protrusion. Also during precursor stabilization the Mena-lamellipodin complex delivers SHIP2 at the precursor core. SHIP2 results in local PI $(3,4) \mathrm{P} 2$ production at the precursor, and this elevated $\mathrm{PI}(3,4) \mathrm{P} 2$ production further stabilizes the invadopodium precursor by lamellipodin-dependent recruitment of Mena ${ }^{\mathrm{INV}}$. Mena ${ }^{\mathrm{INV}}$ inhibits PTP1B thereby maintaining the phosphorylation of cortactin at Y421, which pushes the reactions to the right resulting in actin polymerization as described in Figure 2 leading to invadopodium maturation and matrix degradation. 


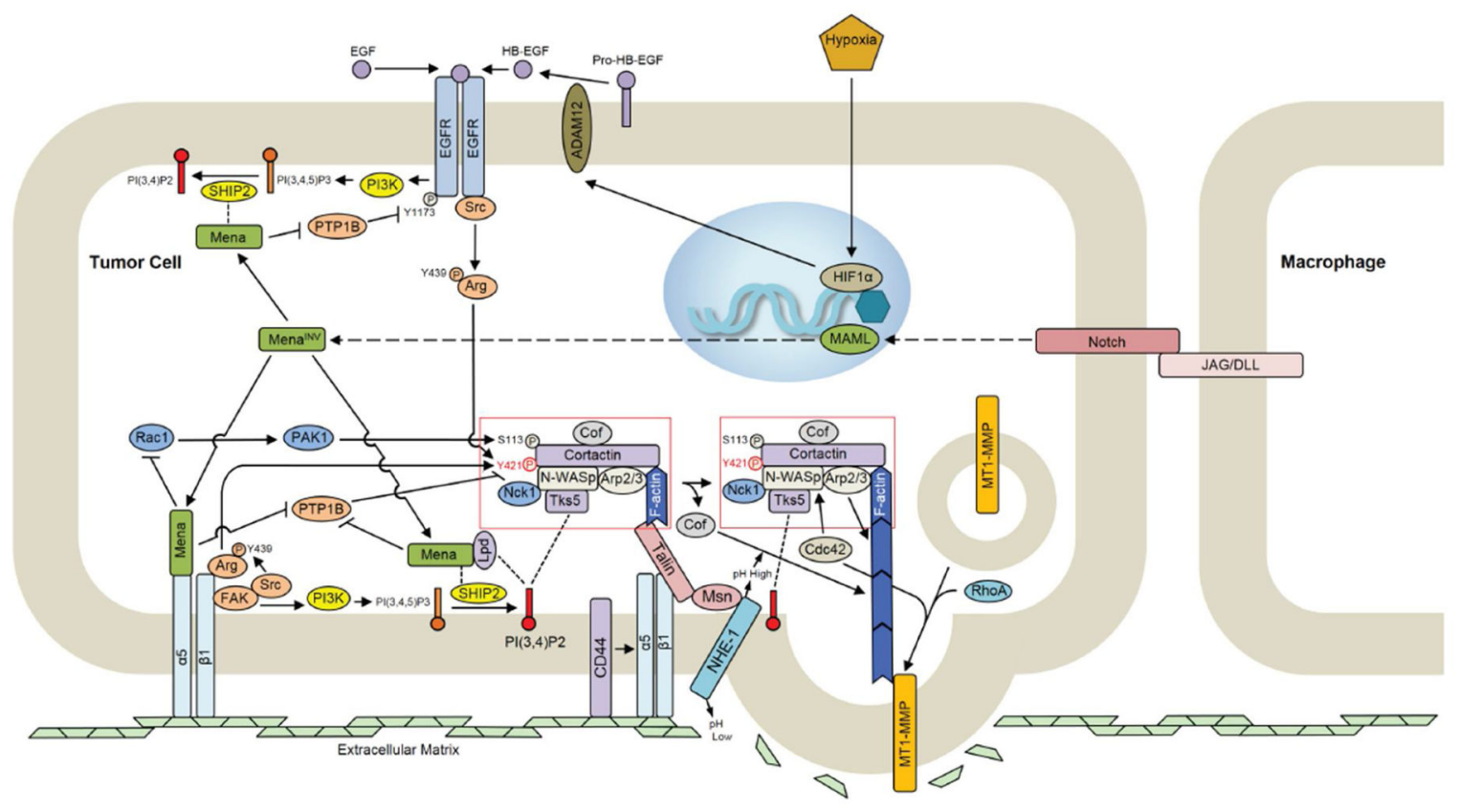

Figure 2. Spatial model showing the pathways involved in initiation of invadopodia from growth factor receptors, ECM and cell-cell contact

Growth factor stimulation and/or ECM-integrin engagement (Table 1), initiates the coassembly of a complex of Tks5, cortactin, N-WASp, cofilin, Arp2/3 and actin forming the initial invadopodium precursor core (red box). Lamellipodin binds PI(3,4)P2 at invadopodium precursor, a lipid that is uniquely localized to the core compared to other phosphoinositides, and recruits Mena isoforms, SHIP2 and Arg kinase to the precursor. SHIP2 produces additional PI $(3,4) \mathrm{P} 2$ to which Tks5 binds thereby stabilizing the precursor by binding it to the plasma membrane. Mena and Arg kinase are also delivered to the core by $\alpha 5$ and $\beta 1$ integrins, respectively. Arg phosphorylates cortactin at Y421 in the core leading to talin-moesin-NHE1 complex recruitment that produces a local increase in $\mathrm{pH}$ to activate cofilin-initiated actin polymerization. CD44 also interacts with components of the ECM, such as fibronectin and hyaluronan, and promotes a $5 \beta 1$ integrin activation and cortactin phosphorylation at invadopodia. Cortactin tyrosine phosphorylation also recruits Nck1. Nck1 and Cdc42 activate the N-WASp-Arp2/3 complex which nucleates actin polymerization from the cofilin-generated $\mathrm{F}$-actin resulting in invadopodium protrusion and maturation. Both Cdc42 and RhoA also promote recruitment of MT1-MMP to the plasma membrane at invadopodia. Mena ${ }^{\mathrm{INV}}$ inhibits PTP1B dephosphorylation of cortactin Y421 thereby supporting the above events and stabilizing invadopodium maturation. In addition, Mena inhibits Rac1-induced invadopodium disassembly by PAK1 phosphorylation of cortactin at S113. Tumor hypoxia has also been shown to promote invadopodium maturation in a Notch-dependent manner via HIF1a-mediated upregulation of ADAM12, which cleaves of HB-EGF from the plasma membrane to activate the EGFR. Macrophage-induced Notch signaling in the tumor cell promotes Mena ${ }^{\mathrm{INV}}$ expression. Mena ${ }^{\mathrm{INV}}$ may form tetramers with other Mena isoforms at RTKs, a 5 integrin and in the invadopodium core. Mena ${ }^{\text {INV }}$ stimulates invadopodium maturation by inhibiting the dephosphorylation of RTKs and cortactin Y421 thereby synergizing with growth factor and ECM signals. FAK activation of 
Src kinase activates Arg kinase acts as described above for growth factor and ECM signaling. FAK activation induces PI3K-dependent generation of PI $(3,4,5) \mathrm{P} 3$ from $\mathrm{PI}(4,5) \mathrm{P} 2$, which is then dephosphorylated by SHIP2 to form PI $(3,4) \mathrm{P} 2$ which binds to Tks5 to stabilize the precursor core. Note that the overlap of the integrin and receptor tyrosine signaling pathways relative to the common invadopodium core structure can also define the crosstalk between growth factor receptors and ECM signals leading to more nuanced invadopodium initiation and function. The models in Figures 1 and 2 are derived from work with rat, mouse and human tumor cell lines and primary breast tumor cells derived from patient biopsies all of which are adenocarcinoma cells. The extent to which these models apply to other types of cancer is unknown. 
Table 1

Stimuli promoting the initiation and maturation of invadopodia.

\begin{tabular}{|c|c|c|c|c|}
\hline Type of Signal & Signal & Primary receptor & Effect & Ref \\
\hline \multirow[t]{7}{*}{ Soluble } & EGF & EGFR (Her1) & $\begin{array}{l}\text { Invadopodium precursor formation and } \\
\text { maturation }\end{array}$ & {$[7,36]$} \\
\hline & $\begin{array}{l}\text { Platelet-derived growth factor } \\
\text { (PDGF) }\end{array}$ & PDGFRa & $\begin{array}{l}\text { Invadopodium precursor formation and } \\
\text { maturation }\end{array}$ & [29] \\
\hline & Tumor Growth Factor- $\beta$ & TGF $\beta$ R1 & $\begin{array}{l}\text { Invadopodium precursor formation and } \\
\text { maturation }\end{array}$ & [28] \\
\hline & $\begin{array}{l}\text { Vascular Endothelial Growth } \\
\text { Factor (VEGF) }\end{array}$ & VEGFR & $\begin{array}{l}\text { Invadopodium maturation (MT1-MMP } \\
\text { recruitment) }\end{array}$ & {$[56]$} \\
\hline & $\begin{array}{l}\text { Hepatocyte Growth Factor } \\
\text { (HGF) }\end{array}$ & C-Met & $\begin{array}{l}\text { Invadopodium precursor formation and } \\
\text { maturation }\end{array}$ & [37] \\
\hline & Heparin binding EGF & EGFR & $\begin{array}{l}\text { Invadopodium precursor formation and } \\
\text { maturation }\end{array}$ & {$[33]$} \\
\hline & $\begin{array}{l}\text { Stromal-cell - derived factor } 1 \\
\text { (Sdf-1) }\end{array}$ & CXCR4 & $\begin{array}{l}\text { Invadopodium maturation (MT1-MMP } \\
\text { recruitment) }\end{array}$ & {$[56]$} \\
\hline \multirow[t]{3}{*}{ Extracellular Matrix } & Fibronectin & $a 5 \beta 1$ & $\begin{array}{l}\text { Invadopodium maturation (Arg-induced } \\
\text { cortactin phosphorylation) }\end{array}$ & [40] \\
\hline & Collagen I (fibrillary) & $\begin{array}{l}\text { Discoid Domain } \\
\text { Receptor (DDR) } \\
\text { a2 } \beta 1\end{array}$ & $\begin{array}{l}\text { Linear invadosome formation and } \\
\text { maturation } \\
\text { Invadopodium formation }\end{array}$ & {$[41,65,66]$} \\
\hline & Collagen, hyaluronan, & $\mathrm{CD} 44 \mathrm{~s}$ & $\begin{array}{l}\text { Invadopodium precursor formation and } \\
\text { maturation }\end{array}$ & {$[52]$} \\
\hline Cell-cell contact & Jagged or DII4 & Notch1 & Invadopodium maturation & {$[16,33]$} \\
\hline
\end{tabular}

